\title{
Dermatite atópica em idade pediátrica - Inibidores da calcineurina ou corticosteróides, qual a melhor opção?
}

Luís Filipe Cavadas,* Vera Pires**

\section{RESUMO}

Objectivo: Comparar a eficácia e a segurança do uso tópico dos inibidores da calcineurina com os corticosteróides no tratamento da dermatite atópica (DA) em idade pediátrica.

Fontes de dados: Base de dados MEDLINE e sítios na internet de medicina baseada na evidência.

Métodos de revisão: Pesquisa de revisões baseadas na evidência, normas de orientação clínica, meta-análises, revisões sistemáticas e ensaios clínicos controlados e aleatorizados, utilizando os termos MeSH:Dermatitis, Atopic; Tacrolimus; Pimecrolimus. Limitou-se a pesquisa a artigos publicados desde Janeiro de 2000 até Agosto de 2009, em Inglês, Francês, Espanhol e Português, restrita aos pacientes dos zero aos 18 anos de idade. Para a comparação de resultados foram usadas escalas internacionais: Investigator's Global Assessment, Eczema Area and Severity Índex e Children's Dermatology Life Quality Índex. Para avaliar o nível de evidência, foi utilizada a escala de Strength of Recommendation Taxonomy (SORT) da American Family Physician.

Resultados: Foram obtidos 76 estudos, tendo apenas dois cumpridos os critérios de inclusão (uma norma de orientação clínica e uma metanálise).

Eficácia: O uso de tacrolimus parece ser mais eficaz no tratamento da DA moderada a grave comparativamente ao acetato de hidrocortisona a $1 \%$. Na DA grave ou muito grave o uso de acetato de metilprednisolona a $0,1 \%$ mostrou ser superior.

Segurança: o uso de tacrolimus esteve associado a maior número de efeitos adversos locais.

Os estudos com pimecrolimus não cumpriram os critérios de inclusão.

Conclusões: Da evidência disponível, o tacrolimus é mais eficaz que o acetato de hidrocortisona a $1 \%$ no tratamento da DA moderada a grave (SOR B).

Na exacerbação grave ou muito grave da DA o uso de aceponato de metilprednisolona $0,1 \%$ mostrou ter eficácia superior ao tacrolimus (SOR A).

Palavras-chave: Dermatite Atópica; Tacrolimus; Pimecrolimus; Criança; Adolescente.

\section{INTRODUÇÃO}

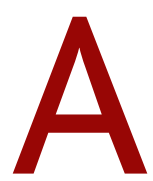
dermatite atópica (DA) é uma patologia inflamatória crónica que se caracteriza por lesões eczematosas e pruriginosas, cuja localização varia conforme a idade, sendo predominantes na face, nádegas e superfícies extensoras na primeira infância e nas superfícies flexoras posteriormente. ${ }^{1}$ Esta patologia apresenta uma prevalência

*Interno de Medicina Geral e Familiar, Centro de Saúde da Senhora da Hora - Unidade Local de Saúde Matosinhos.

**Interna de Medicina Geral e Familiar, Unidade de Saúde Lordelo do Ouro - ACES Porto Ocidental. mundial estimada de $10-20 \%$ em crianças e $1-3 \%$ nos adultos, sendo que cerca de $60 \%$ dos casos remitem durante a adolescência. ${ }^{2-4}$ Não existem dados consistentes quanto à prevalência da DA em Portugal.

A etiologia desta patologia é desconhecida mas provavelmente dever-se-á à combinação de causas genéticas e ambientais (exposição a alergénios, irritantes e bactérias, baixa classe social, famílias numerosas) e está associada à marcha alérgica, sendo factor de risco para o desenvolvimento de asma. ${ }^{5}$

Quanto à sua fisiopatologia, a DA parece estar relacionada com a activação de linfócitos T-helper tipo 2 , 
levando ao aumento da produção de interleucina 4 que promove a libertação de imunoglobulina $\mathrm{E}$ (IgE).$^{5}$

A DA tem um impacto significativo na qualidade de vida da criança e dos pais. O prurido associado a esta patologia condiciona escoriações e liquenificação da pele, bem como distúrbios do sono e sonolência diurna. Por outro lado, a existência de uma patologia cutânea visível desperta estigma social. Do ponto de vista económico a DA implica um maior número de idas ao médico e o uso continuado de emolientes e fármacos.

Os objectivos do tratamento consistem na redução da inflamação local e dos sintomas associados como o prurido. Durante vários anos o tratamento farmacológico standard da DA ligeira a moderada tem-se baseado na utilização de corticosteróides tópicos. Contudo, quando a sua utilização é prolongada assiste-se a efeitos adversos cutâneos, nomeadamente atrofia, telangiectasias, dermite perioral, rosácea, agravamento da acne e surgimento de estrias violáceas. ${ }^{6,7}$ Alguns estudos referem, ainda, como efeitos sistémicos da corticoterapia prolongada as alterações do crescimento e do desenvolvimento da criança, embora sem evidência conclusiva. ${ }^{5,7,8,9}$

Actualmente, existem outros fármacos imunossupressores, como os inibidores da calcineurina, dos quais fazem parte o tacrolimus e o pimecrolimus, que actuam inibindo uma proteína, a calcineurina, a qual está envolvida na activação dos linfócitos T, reduzindo assim a resposta inflamatória. Estes imunomodeladores tópicos têm um mecanismo de acção diferente dos corticosteróides e poderão ser uma opção no tratamento da DA, especialmente em crianças. ${ }^{10}$ Todavia, a maior parte dos estudos com estes fármacos comparam a sua eficácia e segurança com um placebo e não é claro que sejam mais eficazes e seguros que as terapêuticas já existentes. Por tudo isto é fundamental conhecer a melhor evidência disponível sobre a utilização dos inibidores da calcineurina tópicos em crianças.

Este estudo tem como objectivo determinar a eficácia e a segurança do tratamento tópico com inibidores da calcineurina comparativamente aos corticosteróides, na DA em idade pediátrica.

\section{MÉTODOS}

Foi realizada uma pesquisa nas bases de dados MEDLINE, Guidelines Finder, National Guideline Clearinghouse, Canadian Medical Association Infobase, The
Cochrane Library, DARE, Bandolier, TRIP e InfoPoems, de revisões baseadas na evidência, normas de orientação clínica, meta-análises, revisões sistemáticas e ensaios clínicos controlados e aleatorizados, publicados desde 1 de Janeiro de 2000 até 15 de Agosto de 2009, em Inglês, Francês, Espanhol e Português, utilizando os termos MeSH: Dermatitis, Atopic; Tacrolimus; Pimecrolimus. A pesquisa foi restrita aos pacientes em idade pediátrica (zero aos 18 anos de idade).

Os critérios utilizados para a inclusão dos artigos nesta revisão foram:

- População: crianças e adolescentes com diagnóstico de DA e idade inferior a 18 anos.

- Intervenção: aplicação tópica de um inibidor da calcineurina (tacrolimus ou pimecrolimus) ou de um corticosteróide nas lesões de dermatite atópica.

- Comparação: inibidor da calcineurina tópico (tacrolimus ou pimecrolimus) versus corticosteróide tópico

- Resultados: eficácia (desaparecimento ou melhoria da lesão, redução do prurido, qualidade do sono e qualidade de vida da criança) e segurança (efeitos adversos locais).

Para a comparação dos resultados foram utilizadas as escalas internacionalmente aceites. Assim, para a avaliação inicial, evolução e gravidade da lesão com o tratamento foram usadas as escalas Investigator's Global Assessment (IGA) e Eczema Area and Severity Index (EASI); para avaliar a qualidade de vida da criança foi usado o Children's Dermatology Life Quality Índex (CDLQI). ${ }^{11}$

Para avaliar a qualidade dos estudos e a força de recomendação, foi utilizada a escala de Strength of Recommendation Taxonomy (SORT) da American Family Physician (AFP). ${ }^{12}$ Segundo esta taxonomia, a qualidade do estudo está subdividida em três Níveis de Evidência (Nível de Evidência 1: estudos de boa qualidade, evidência orientada para o doente; Nível de Evidência 2: estudos de qualidade limitada, evidência orientada para o doente; e Nível de Evidência 3: outra evidência) e a Força de Recomendação em três graus (Força de Recomendação A: consistente, evidência orientada para o doente; Força de Recomendação B: inconsistente ou qualidade limitada, evidência orientada para o doente; e Força de Recomendação C: consensos, evidência orientada para a doença). ${ }^{12}$ 


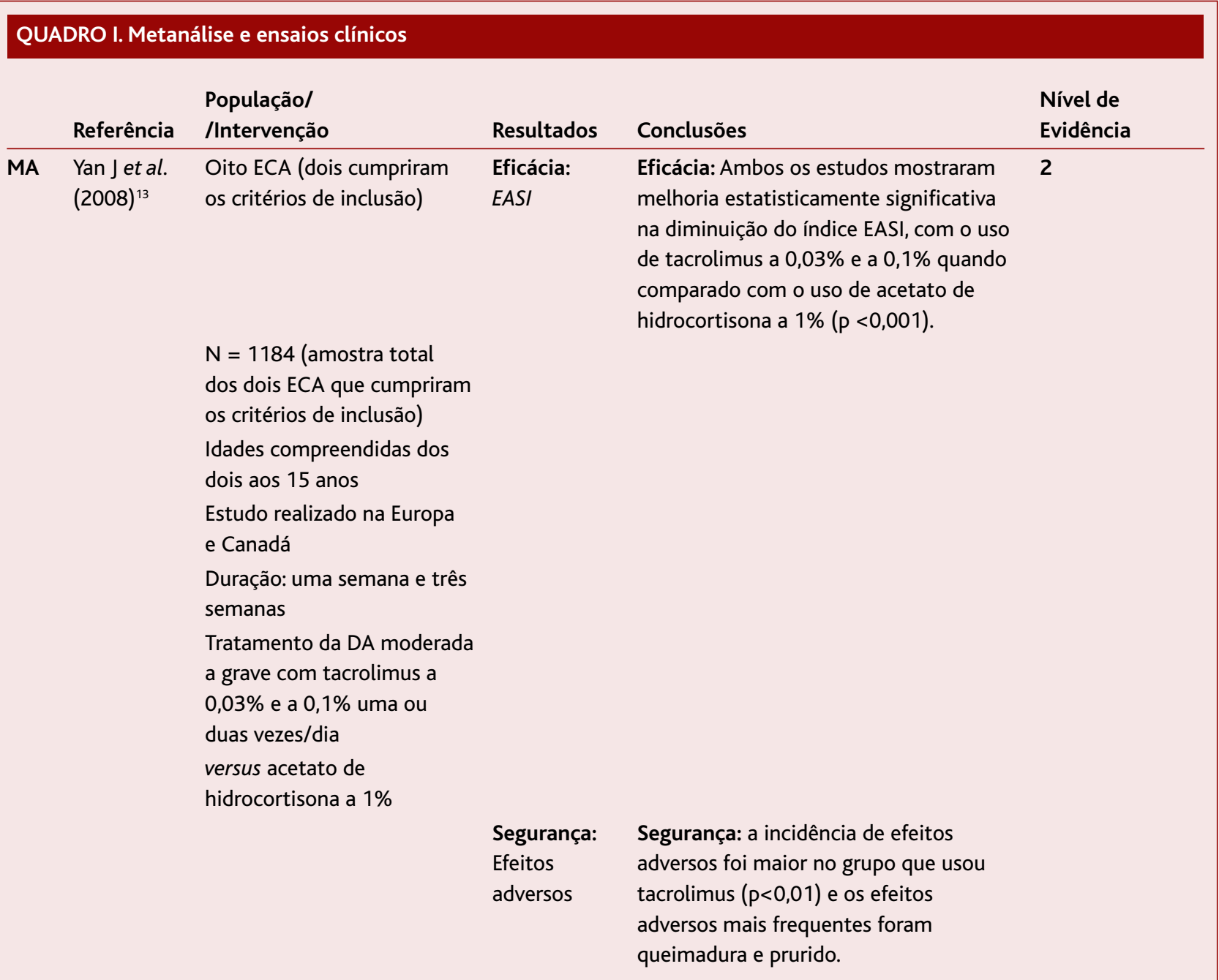

\section{RESULTADOS}

A pesquisa efectuada resultou na identificação de 76 artigos. Destes foram excluídos os artigos repetidos, aqueles em que se verificou discordância com o objectivo da revisão e aqueles que não cumpriam os critérios de inclusão previamente descritos.

Obtiveram-se dois artigos, que foram incluídos nesta revisão: uma norma de orientação clínica (NOC) e uma metanálise.

A NOC incluída apresentou cinco ensaios clínicos atribuindo níveis de evidência próprios, que não têm conversão para a taxonomia SORT. A mesma NOC não conclui com forças de recomendação. Para evitar erros de conversão de taxonomias diferentes os autores ana- lisaram individualmente os estudos apresentados pela NOC. Destes cinco ensaios clínicos dois estavam presentes na metanálise (que foi incluída), dois foram analisados individualmente e um não foi analisado por impossibilidade de obtenção do artigo (Quadro I).

Para uma melhor compreensão, os resultados foram agrupados de acordo com a eficácia e segurança dos inibidores da calcineurina em relação aos corticosteróides.

\section{Eficácia dos inibidores da calcineurina em relação aos corticosteróides}

A metanálise ${ }^{13}$ incluída apresentou oito ensaios clínicos, dois dos quais comparavam o uso dos inibido- 


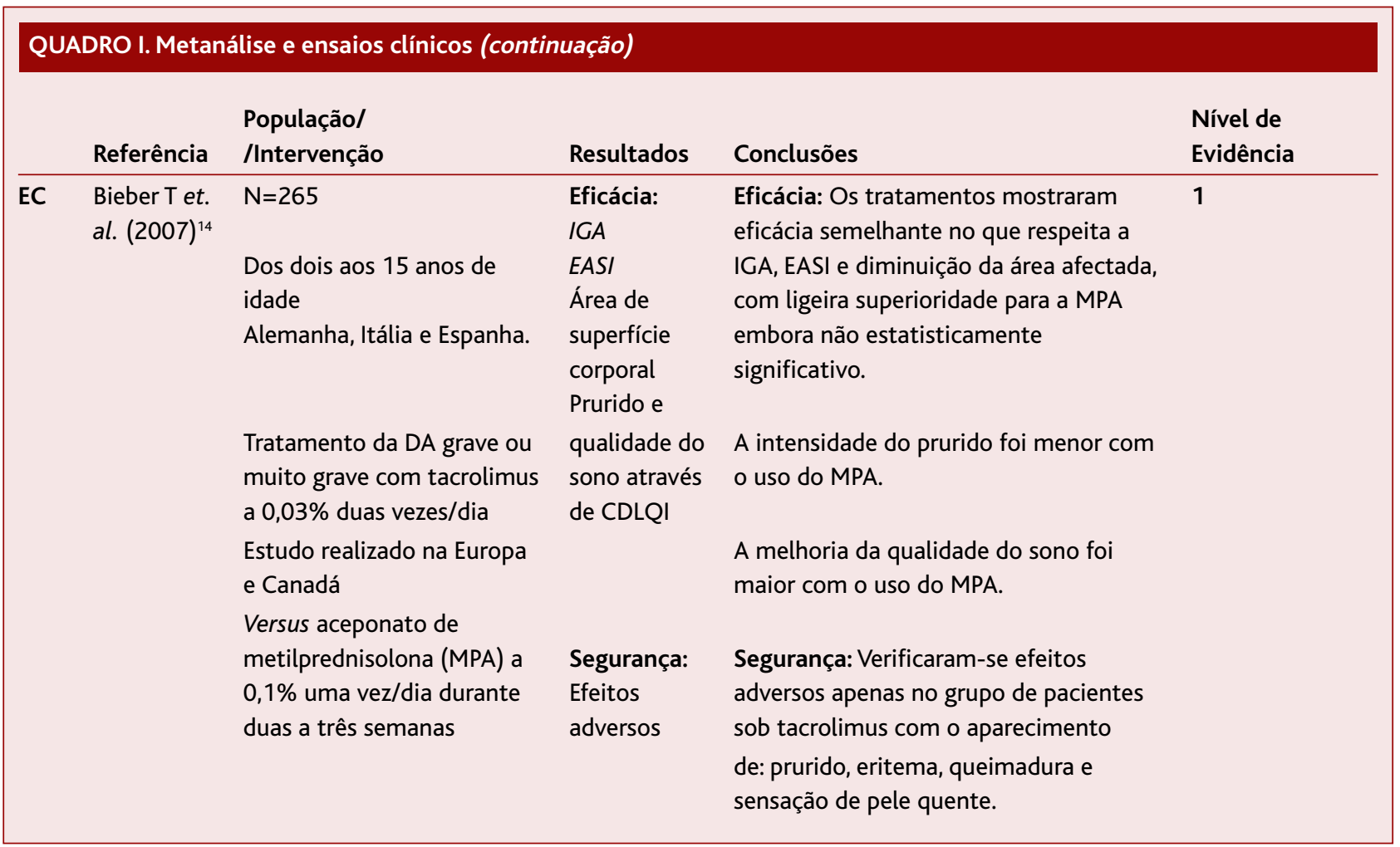

res da calcineurina com corticosteróides, em pacientes dos dois aos 17 anos de idade, com DA moderada a grave segundo a escala de Rajka and Langeland. ${ }^{14} \mathrm{~A}$ escala de Rajka and Langeland categoriza a gravidade da DA em leve, moderada ou grave em função do envolvimento da superfície cutânea, da evolução da doença e do prurido nocturno. Nestes estudos, o uso de tacrolimus foi mais eficaz do que o uso do acetato de hidrocortisona a $1 \%$, com melhoria do EASI. ${ }^{13}$

No ensaio clínico de Bieber T et al, ${ }^{15}$ realizado a pacientes dos dois aos 15 anos de idade, foram incluídas crianças e jovens com exacerbação grave ou muito grave da DA (IGA superior ou igual a 4), ou seja, classificaram o episódio agudo e não a gravidade da doença. Na exacerbação grave ou muito grave da DA, o uso de aceponato de metilprednisolona $0,1 \%$, mostrou ter eficácia superior ao tacrolimus com melhoria no EASI, na diminuição da área de superfície corporal afectada, na diminuição da intensidade do prurido e na melhoria da qualidade do sono.

O ensaio clínico de Arkwright et al ${ }^{16}$ incluiu crianças dos seis meses aos 18 anos com DA moderadamente grave, assim classificada por ser não totalmente controlada com uso regular de corticosteróides ou emolientes, e comparou o uso de tacrolimus e corticosteróides tópicos em simultâneo na mesma criança, mas em lados do corpo diferentes. Os resultados obtidos foram analisados de acordo com a melhoria ou agravamento do eritema das lesões em tratamento e/ou a liquenificação cutânea observada visualmente ou percepcionada ao toque. A utilização do tacrolimus tópico foi mais eficaz do que o uso dos corticosteróides tópicos em $77 \%$ dos casos. A análise multivariada por regressão logística das variáveis idade, sexo, superfície de área afectada pré-tratamento e potência do corticosteróide pré-ensaio, mostrou que o uso tópico de corticosteróides de potência moderada ou potentes foi o único factor que reduziu a possibilidade de se observar um efeito benéfico com o tacrolimus. ${ }^{16}$ Contudo este estudo não especifica qual a escala utilizada na classificação da potência dos corticosteróides.

Não foi incluído nenhum estudo que comparasse a eficácia do uso de pimecrolimus com um corticosteróide. 


\begin{tabular}{|c|c|c|c|c|c|}
\hline & Referência & $\begin{array}{l}\text { População/ } \\
\text { /Intervenção }\end{array}$ & Resultados & Conclusões & $\begin{array}{l}\text { Nível de } \\
\text { Evidência }\end{array}$ \\
\hline EC & $\begin{array}{l}\text { Arkwright, } \\
\text { et al. } \\
(2007)^{15}\end{array}$ & $\begin{array}{l}\mathrm{n}=96 \\
\text { Dos seis meses aos } 18 \text { anos } \\
\text { Tratamento da DA } \\
\text { moderadamente grave com } \\
\text { tacrolimus a } 0,03 \% \text { e a } \\
\text { 0,1\% duas vezes/dia versus } \\
\text { corticosteróide tópico que } \\
\text { já utilizavam regularmente } \\
\text { (hidrocortisona a } 1 \% \text {, } \\
\text { acetonido de fluocinolona } \\
\text { a 0,00625\%, butirato de } \\
\text { clobetasona a } 0,05 \% \text {, } \\
\text { valerato de betametasona } \\
\text { a } 0,025 \%, \text { butirato de } \\
\text { hidrocortisona a } 0,1 \% \text {, } \\
\text { furoato de mometasona a } \\
0,1 \% \text { ). Tacrolimus aplicado } \\
\text { num lado do corpo e } \\
\text { corticosteróide no outro } \\
\text { lado com comparação } \\
\text { directa na mesma criança. }\end{array}$ & $\begin{array}{l}\text { Eficácia: } \\
\text { Eritema e/ou } \\
\text { liquenificação } \\
\text { da pele } \\
\text { visualizável } \\
\text { ou ao toque } \\
\text { avaliados } \\
\text { pelo } \\
\text { observador. }\end{array}$ & $\begin{array}{l}\text { Eficácia: A utilização do tacrolimus } \\
\text { tópico foi mais eficaz do que o uso dos } \\
\text { corticosteroides tópicos em } 77 \% \text { dos } \\
\text { casos. A análise multivariada por } \\
\text { regressão logística das variáveis idade, } \\
\text { sexo, superfície de área afectada } \\
\text { pré-tratamento e potência do } \\
\text { corticosteróide pré-ensaio, mostrou que } \\
\text { o uso tópico de corticosteróides de } \\
\text { potência moderada ou potentes foi o } \\
\text { único factor que reduziu a possibilidade } \\
\text { de se observar um efeito benéfico com } \\
\text { o tacrolimus. } \\
\text { Não especifica qual a escala utilizada } \\
\text { na classificação da potência dos } \\
\text { corticosteróides. }\end{array}$ & 2 \\
\hline
\end{tabular}

Legenda: MA - Metanálise, EC - Ensaio clínico, n - número amostral, ECA - Estudo controlado e aleatorizado, EASI - Eczema Area and Severity Índex, IGA - Investigator's Global Assessment, CDLQI - Children's Dermatology Life Quality Índex, MPA - aceponato de metilprednisolona.

\section{Segurança dos inibidores da calcineurina em relação aos corticosteróides}

Quer na metanálise, ${ }^{13}$ quer no ensaio clínico de Bieber $\mathrm{T}$ et $a l,{ }^{15} \mathrm{o}$ uso de tacrolimus esteve associado a maior número de efeitos adversos locais, nomeadamente prurido, eritema e queimadura.

Não foi incluído nenhum estudo que comparasse a segurança do uso de pimecrolimus com um corticosteróide.

Verificou-se que os estudos apresentaram como fontes de financiamento empresas farmacológicas ligadas à produção e comercialização dos inibidores da calcineurina (Quadro II).

\section{CONCLUSÕES}

Os estudos que compararam os inibidores da calcineurina com os corticosteróides foram realizados em crianças com mais de dois anos de idade e apenas o estudo de Arkwright, et al ${ }^{16}$ foi realizado em crianças com mais de seis meses de idade.

Não foram analisados os estudos com pimecrolimus por não cumprirem os critérios de inclusão.

Os estudos incluídos apresentaram diferentes definições para a gravidade da DA, diferentes concentrações de tacrolimus $(0,03 \%$ e $0,1 \%)$ e corticosteróides diferentes.

No que diz respeito ao financiamento e elaboração 


\begin{tabular}{|c|c|c|c|}
\hline Referência & $\begin{array}{l}\text { Conflito de } \\
\text { interesses }\end{array}$ & $\begin{array}{l}\text { Fontes de } \\
\text { financiamento }\end{array}$ & Observações \\
\hline $\begin{array}{l}\text { NICE } \\
(2007)^{10}\end{array}$ & Consta & Não consta & $\begin{array}{l}\text { Na sua elaboração intervêm profissionais de saúde de diferentes áreas } \\
\text { médicas (pediatras, dermatologistas, enfermeiros, farmacêuticos, } \\
\text { economistas, entre outros), nos conflitos de interesses consta a indústria } \\
\text { farmacêutica ligada aos inibidores da calcineurina. }\end{array}$ \\
\hline $\begin{array}{l}\text { Yan J et al. } \\
(2008)^{13}\end{array}$ & Não consta & Não consta & $\begin{array}{l}\text { Os oito estudos foram patrocinados pela Fujisawa. Os autores assumem } \\
\text { provável viés neste estudo. }\end{array}$ \\
\hline $\begin{array}{l}\text { Bieber T et al. } \\
(2007)^{14}\end{array}$ & Não consta & $\begin{array}{l}\text { Intendis } \\
\text { GmbH, Berlin }\end{array}$ & \\
\hline $\begin{array}{l}\text { Arkwright, et al. } \\
(2007)^{15}\end{array}$ & Não consta & Não consta & \\
\hline
\end{tabular}

dos estudos, mesmo quando as fontes de financiamento não constavam no artigo, como no caso da norma de orientação clínica, verificou-se que a indústria farmacêutica participou em todos os estudos analisados. Assim sendo, o facto das companhias farmacêuticas responsáveis pela produção dos inibidores da calcineurina dirigirem a totalidade dos estudos aumenta a preocupação acerca de potenciais viéses.

Um dos estudos apresentados pela NOC não foi analisado por impossibilidade de obtenção do artigo, podendo introduzir um viés de informação.

Outros dois potenciais viéses são a não inclusão de outros descritores, o que poderia ter aumentado o número de artigos incluídos, bem como o intervalo temporal de pesquisa definido pelos autores.

Em conclusão, e de acordo com os resultados obtidos, pode-se afirmar que, em crianças com mais de dois anos de idade:

- O tacrolimus é mais eficaz que o acetato de hidrocortisona a $1 \%$, no tratamento da DA moderada a grave, classificada segundo a escala de Rajka and Langeland ${ }^{14}$ (SOR B).

- Na exacerbação grave ou muito grave da DA (IGA superior ou igual a 4) o uso de aceponato de metilprednisolona $0,1 \%$ mostrou ter eficácia superior ao tacrolimus (SOR A).

São necessários estudos controlados e aleatorizados, de longa duração e de elevada qualidade, que comparem estes fármacos, nas suas diferentes concentrações, com uma maior diversidade de corticosteróides e em idade pediátrica compreendida desde os zero e os 18 anos de idade. É fundamental a uniformização dos estudos, com a utilização da mesma definição de gravidade da DA para possíveis comparações. Todos estes estudos devem ser criteriosamente realizados, devendo conter, sempre, uma declaração de conflito de interesses e o financiamento deve ser proveniente de fontes isentas.

\section{REFERÊNCIAS BIBLIOGRÁFICAS}

1. Leung DY, Bieber T. Atopic dermatitis. Lancet 2003 Jan 11; 361 (9352): 151-60.

2. Kay J, Gawkrodger DJ, Mortimer MJ, Jaron AG. The prevalence of childhood atopic eczema in a general population. J Am Acad Dermatol 1994 Jan; 30 (1): 35-9.

3. Spergel JM, Paller AS. Atopic dermatitis and the atopic march. J Allergy Clin Immunol 2003 Dec; 112 (6 Suppl): S118-27.

4. Larsen FS, Hanifin JM. Epidemiology of atopic dermatitis. Immunol Allergy Clin North Am 2002 Feb; 22 (1) :1-24.

5. Buys L. Treatment options for atopic dermatitis. Am Fam Physician 2007 Feb 15; 75 (4): 523-8, 530.

6. Su JC, Kemp AS, Varigos GA, Nolan TM. Atopic eczema: its impact on the family and financial cost. Arch Dis Child 1997 Feb; 76 (2): 159-62.

7. Ference JD, Last AR. Choosing Topical Corticosteroids. Am Fam Physician 2009 Jan 15; 79 (2):135-40.

8. Williams HC. Atopic eczema. BMJ 1995 Nov 11; 311 (6983): 1241-2.

9. Bergmann RL, Edenharter G, Bergmann KE, Forster J, Bauer CP, Wahn V, et al. Atopic dermatitis in early infancy predicts allergic airway disease at 5 years. Clin Exp Allergy 1998 Aug; 28 (8): 965-70.

10. National Institute for Health and Clinical Excellence. Atopic eczema in children: management of atopic eczema in children from birth up to 
the age of 12 years. London: NICE; 2007. Disponível em: http://guidance.nice.org.uk/CG57/Guidance/pdf/English [acedido em 25/07/2009].

11. Garside R, Stein K, Castelnuovo E, Pitt M, Ashcroft D, Dimmock P, et al. The effectiveness and cost-effectiveness of pimecrolimus and tacrolimus for atopic eczema: a systematic review and economic evaluation. Health Technol Assess 2005 Jul; 9 (29): iii, xi-xiii, 1-230.

12. Ebell MH, Siwek J,Weiss BD,Woolf SH, Susman J, Ewingman B, et al. Strength of Recommendation Taxonomy (SORT): a patient-centered approach to grading evidence in the medical literature. Am Fam Physician 2004 Feb 1; 69 (3): 548-56.

13. Yan J, Chen SL, Wang XL, Zhou W, Wang FS. Meta-analysis of tacrolimus ointment for atopic dermatitis in pediatric patients. Pediatr Dermatol 2008 Jan-Feb; 25 (1): 117-20.

14. Rajka G, Langeland T. Grading of the severity of atopic dermatitis. Acta Dermatol Venereol Suppl (Stockh) 1989; 144: 13-4.

15. Bieber T,Vick K, Fölster-Holst R, Belloni-Fortina A, Städtler G, Worm M, et al. Efficacy and safety of methylprednisolone aceponate ointment $0.1 \%$ compared to tacrolimus $0.03 \%$ in children and adolescents with an acute flare of severe atopic dermatitis.Allergy. 2007 Feb; 62 (2): 1849.

16. Arkwright PD, Gillespie MC, Ewing Cl, David TJ. Blinded side-to-side comparison of topical corticosteroid and tacrolimus ointment in children with moderate to severe atopic dermatitis. Clin Exp Dermatol 2007 Mar; 32 (2): 145-7.

\section{CONFLITOS DE INTERESSE}

Os autores declaram não existir conflitos de interesse na elaboração deste artigo.

\author{
ENDEREÇO PARA CORRESPONDÊNCIA \\ Luís Filipe Cavadas, \\ Centro de Saúde da Senhora da Hora, Rua da Lagoa, s/n. ${ }^{\circ}$ \\ 4460 Senhora da Hora \\ E-mail: luisfilipemcavadas@gmail.com
}

Recebido em 25/11/2009

Aceite para publicação em 18/06/2010

\section{ABSTRACT}

\section{ATOPIC DERMATITIS IN CHILDREN - CALCINEURIN INHIBITORS OR CORTICOSTEROIDS, WHAT IS THE BEST OPTION?}

Objective: To compare the efficacy and safety of topical use of calcineurin inhibitors with corticosteroids in the treatment of atopic dermatitis (AD), in children.

Data sources: MEDLINE database and evidence-based medicine websites.

Review methods: The authors searched evidence-based reviews, guidelines, meta-analysis, systematic reviews and randomized controlled trials, using the MeSH terms: Dermatitis, Atopic; Tacrolimus, Pimecrolimus. The search was limited to articles published between January 2000 and August 2009, in English, French, Spanish and Portuguese, restricted to patients from zero to 18 years of age. International scales were used to describe the results: Investigator's Global Assessment, Eczema Area and Severity Index and Children's Dermatology Life Quality Index. Recommendation Taxonomy (SORT) of the American Family Physician was used to assess the level of evidence.

Results: One guideline and one meta-analysis met the inclusion criteria from 76 studies obtained.

Efficacy: The use of tacrolimus appears to be more effective in the treatment of moderate to severe AD, when compared to $1 \%$ hydrocortisone acetate. In the severe to very severe $A D$ the use of $0,1 \%$ methylprednisolone aceponate have demonstrated to be superior.

Safety: the use of tacrolimus was associated with more local adverse effects.

Studies with pimecrolimus failed the inclusion criteria.

Conclusions: From the available evidence Tacrolimus is more effective than $1 \%$ hydrocortisone acetate in the treatment of moderate to severe AD (SOR B).

In severe or very severe exacerbation of $A D$ the use of $0,1 \%$ methylprednisolone aceponate proved to be superior than tacrolimus (SOR A).

Keywords: Dermatitis, Atopic; Tacrolimus; Pimecrolimus; Child; Adolescent. 